\title{
Striatal Dopamine Mediates the Interface between Motivational and Cognitive Control in Humans: Evidence from Genetic Imaging
}

\author{
Esther Aarts*',2, Ardi Roelofs', Barbara Franke' ${ }^{1,2,3}$, Mark Rijpkema', Guillén Fernández ${ }^{1,4}$, \\ Rick C Helmich ${ }^{1,4,5}$ and Roshan Cools ${ }^{1,2}$ \\ 'Donders Institute for Brain, Cognition and Behaviour, Radboud University Nijmegen, Nijmegen, The Netherlands; ${ }^{2}$ Department of Psychiatry, \\ Radboud University Nijmegen Medical Centre, Nijmegen, The Netherlands; ${ }^{3}$ Department of Human Genetics, Radboud University Nijmegen \\ Medical Centre, Nijmegen, The Netherlands; ${ }^{4}$ Department of Neurology, Radboud University Nijmegen Medical Centre, Nijmegen, \\ The Netherlands; ${ }^{5}$ Parkinson Centre Nijmegen (ParC), Radboud University Nijmegen Medical Centre, Nijmegen, The Netherlands
}

\begin{abstract}
Dopamine has been hypothesized to provide the basis for the interaction between motivational and cognitive control. However, there is no evidence for this hypothesis in humans. We fill this gap by using $\mathrm{AMRI}$, a novel behavioral paradigm and a common polymorphism in the DATI gene (SLC6A3). Carriers of the 9-repeat (9R) allele of a 40 base pair repeat polymorphism in the $3^{\prime}$ untranslated region of $D A T I$, associated with high striatal dopamine, showed greater activity in the ventromedial striatum during reward anticipation than homozygotes for the 10-repeat allele, replicating previous genetic imaging studies. The crucial novel finding is that $9 R$ carriers also exhibited a greater influence of anticipated reward on switch costs, as well as greater activity in the dorsomedial striatum during task switching in anticipation of high reward relative to low reward. These data establish a crucial role for human striatal dopamine in the modulation of cognitive flexibility by reward anticipation, thus, elucidating the neurochemical mechanism of the interaction between motivation and cognitive control.

Neuropsychopharmacology (2010) 35, 1943-1951; doi:I0.1038/npp.2010.68; published online 12 May 2010
\end{abstract}

Keywords: DAT polymorphism; SLC6A3; fMRI; ventral striatum; reward; task switching

\section{INTRODUCTION}

Mesolimbic dopamine has long been implicated in reward and motivation. Specifically, animal studies have highlighted a role of dopamine in the ventral striatum, an important structure in the mesolimbic reward pathway, in preparatory responding to signals that predict reward (see Baldo and Kelley, 2007; Berridge and Robinson, 1998; Ikemoto and Panksepp, 1999; Robbins and Everitt, 1992; Schultz, 2002). One of the behavioral consequences of such reward-predictive signal processing is increased exploration of different responses in search of that reward. Such exploration benefits from a variable, flexible behavioral repertoire, and contrasts with the relatively inflexible or stereotypical behaviors that occur during the receipt of reward (Baldo and Kelley, 2007; Craig, 1918). The aim of this study is to show this influence of reward anticipation

*Correspondence: Dr E Aarts, Donders Centre for Cognitive Neuroimaging, Radboud University Nijmegen, P.O. Box 9101, 6500 HB Nijmegen, The Netherlands, Tel: + 3124 36/4793, Fax: + 3124 3610989, E-mail: e.aarts@donders.ru.nl

Received 6 November 2009; revised I March 20 10; accepted 9 April 2010 on flexibility in the domain of human cognitive control and to elucidate its neurobiological mechanism.

Work with behaving experimental animals hints at the importance of dopamine in the striatum for such interfacing between motivation, cognition, and action (Baldo and Kelley, 2007; Mogenson et al, 1980). In keeping with this hypothesis, post-mortem data from non-human primates have revealed an arrangement of spiraling connections between the midbrain and the striatum that seems perfectly suited to subserve a mechanism by which dopamine can direct information flow from ventromedial, through central, to dorsolateral striatal regions (Haber et al, 2000) (see also Nauta et al, 1978). Accordingly, we hypothesized that the interaction between motivation and cognitive flexibility is mediated by dopamine in the striatum.

One approach to studying the role of dopamine in humans is by investigating the effects of genetic variation in dopamine transmission. For instance, variants of the dopamine transporter (DAT) gene, known to affect striatal dopamine, have been observed to predict reward-related activity in the ventral striatum (Dreher et al, 2009; Forbes et al, 2009). In this event-related fMRI study, we adopted this approach to assess dopamine-dependent effects of 
reward on task switching by stratifying our effects by interindividual variation in the $40 \mathrm{bp}$ variable number of tandem repeats polymorphism in the $3^{\prime}$ untranslated region of the DAT gene (DAT1, SLC6A3). The 10-repeat (10R) allele has been associated with increased gene expression and presumably lower levels of synaptic dopamine in the striatum relative to the 9-repeat (9R) allele (eg Fuke et al, 2001; Heinz et al, 2000; Mill et al, 2002; but see van Dyck et al, 2005).

A task-switching design was used with reward-cues preceding each trial and feedback after each response on incongruent arrow-word targets (Figure 1). The taskswitching design is one of the best established paradigms for assessing cognitive flexibility (Monsell, 2003; Sakai, 2008). Showing that dopamine in the striatum mediates the interface between motivation and cognitive flexibility would extend current theoretical models of striatal dopamine, which so far have focused almost exclusively on its role in the habitual control of behavior and reinforcement learning (Daw et al, 2005; Dayan, 2008). We show that striatal dopamine is critical also for higher-order flexible cognitive control processes that are directly sensitive to changes in motivational state.

\section{MATERIALS AND METHODS}

\section{Participants}

Twenty neurologically healthy Dutch undergraduates (10 female and 10 male, mean age 21.6 years, range 18-27) participated in the experiment. All participants were right handed and native speakers of Dutch. Participants were screened for psychiatric, neurological, and vascular disorders, claustrophobia, and metal parts in body. They were compensated for participation, and gave written informed consent in a manner approved by the local ethics committee on research involving human participants.

\section{Genotyping}

Methods of DNA extraction from the saliva samples and genotyping are described in Supplementary Methods.

For DAT1, two genotype groups were established: 11 participants (55\% female, mean age: 21.6 ) were homozygous for the common $10 \mathrm{R}$ allele (10R homozygotes) and 9 participants (44\% female, mean age: 22.4$)$ were heterozygous for the $9 \mathrm{R}$ allele (9R carriers). We also genotyped the catechol-O-methyltransferase gene COMT rs4680 $\left(\mathrm{Val}^{108 / 158} \mathrm{Met}\right.$ ) single nucleotide polymorphism. For COMT, participants were classified as having two (Met/Met; $n=2$ ), one (Val/Met; $n=13$ ), or no Met-alleles (Val/Val; $n=5$ ). The DAT1 10R/10R group consisted of two Met/Met participants, seven Val/Met participants, and two Val/Val participants. The DAT1 9R/10R group consisted of six Val/ Met participants and three Val/Val participants.

\section{Task}

Participants were scanned while performing a pre-cued task-switching task (Figure 1). The targets to which

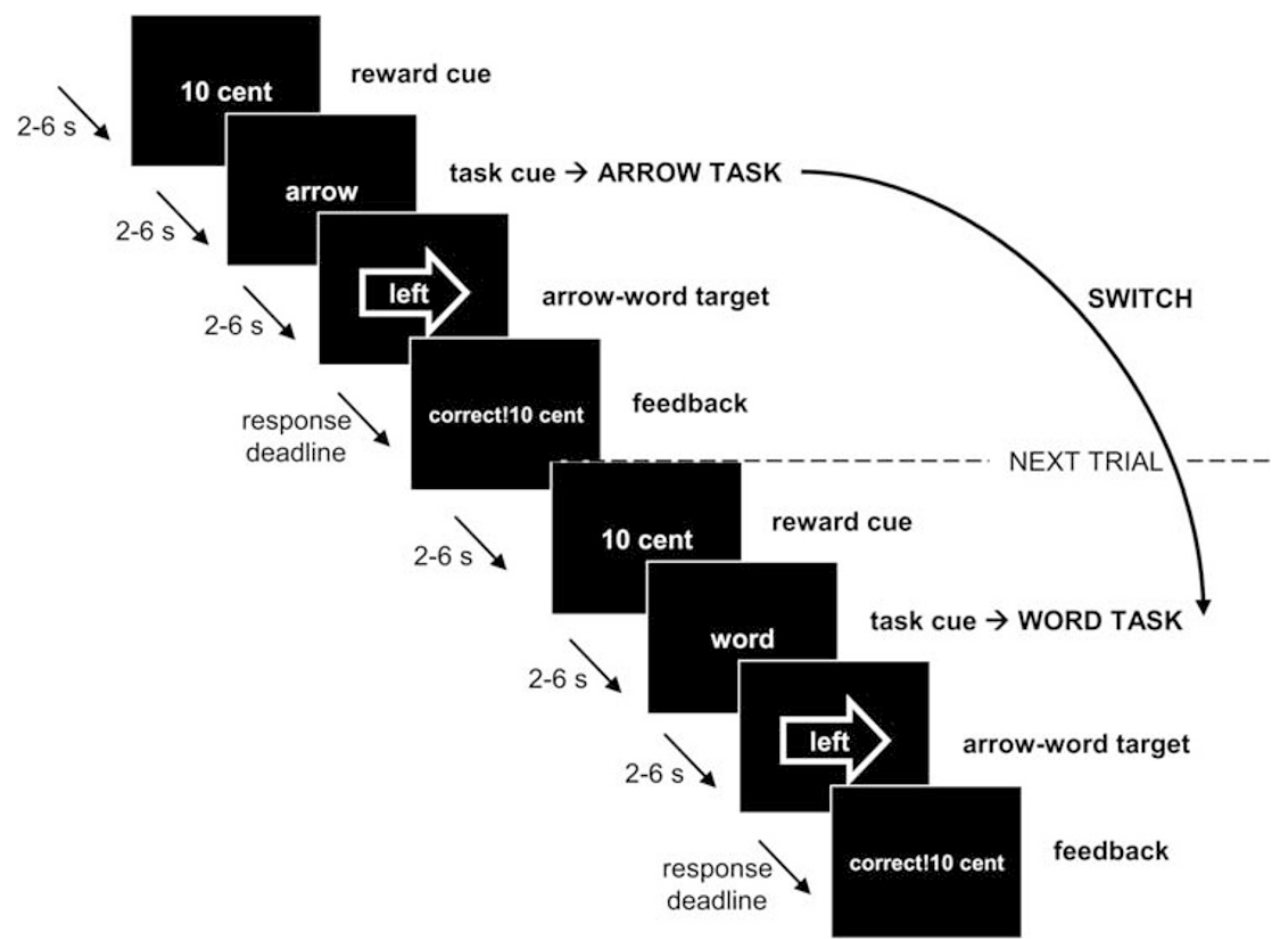

Figure I Example trials from the experimental paradigm. In both of these trials, the reward-cue indicated that the participant could earn 10 cents with a correct and sufficiently quick response (as opposed to I cent in the low-reward condition). The task-cue told the participant to respond to the arrow of the incongruent arrow-word stroop-like target in the first trial, but to the word of the incongruent arrow-word stroop-like target in the second trial. Hence, the second trial is an example of a switch of the task relative to the previous trial. Immediately after the response with a button box, feedback was given with the amount of reward the participant had earned for this specific trial. There was a variable delay of 2-6s between cues and targets in which participants had to fixate on an asterisk in the middle of the screen. 
participants had to respond were incongruent arrow-word combinations (see also Aarts et al, 2008, 2009; Roelofs et al, 2006). Participants responded either to the direction of the arrow (arrow task) or to the direction indicated by the word (word task), which were always in conflict. A task-cue indicated which task to perform. The cues for the arrow task were the Dutch words for arrow (pijl) and shape (vorm). The cues for the word task were the Dutch words for word (woord) and letter (letter). Compared with the previous trial, the task-cue switched on every trial, whereas the task itself switched (from arrow to word or vice versa) or was repeated (from arrow to arrow or from word to word) in a random manner. This way, a task switch (ie the manipulation of interest) was never confounded with a task-cue switch (Logan and Bundesen, 2003). The critical measure of interest, the switch cost, was calculated by subtracting performance (errors and reaction times) on repeat trials from that on switch trials.

In addition, a reward-cue (reward anticipation) preceded each task-cue, telling the participants whether 1 cent (low reward) or 10 cents (high reward) could be earned with a correct and quick response, denoted by the words 1 cent or 10 cent. After the participant's response, feedback was given (reward receipt). Positive feedback was given for a correct response and depended on the preceding reward-cue at the beginning of the trial: correct! 1 cent or correct! 10 cents. Negative feedback was given for an incorrect response (wrong! 0 cent) or a missed response (too late! 0 cent). The feedback for correct responses was given in green, the feedback for incorrect responses in red, and the feedback for misses in blue.

The main experiment consisted of 160 trials. The factors reward (high/low), task (arrow/word), trial type (switch/ repeat), and response (right/left) were equally distributed over the trials in a random manner. The whole experiment lasted for about $40 \mathrm{~min}$ with a $30 \mathrm{~s}$ break after every 32 trials. In the break, the amount of money the participant had earned thus far in the experiment was displayed on the screen.

\section{fMRI Data Acquisition}

Whole-brain imaging was performed on a $3 \mathrm{~T}$ MR scanner (Magnetom Trio Tim, Siemens Medical Systems, Erlangen, Germany). Functional data were acquired using a gradientecho echo-planar scanning sequence (30 axial slices, repetition time $=2020 \mathrm{~ms}$, echo time $=31 \mathrm{~ms}$, voxel size $=3.5 \times 3.5 \times 3.0 \mathrm{~mm}$, interslice gap $=0.5 \mathrm{~mm}$, field of view $=224 \mathrm{~mm}$, flip angle $=80^{\circ}$ ). All functional images were acquired in a single run lasting $\sim 40 \mathrm{~min}$. Visual stimuli were projected on a screen and were viewed through a mirror attached to the head coil. Before the acquisition of functional images, a high-resolution T1-weighted MP-RAGE anatomical scan was obtained (192 saggital slices, repetition time $=2300 \mathrm{~ms}$, echo time $=3.03 \mathrm{~ms}$, voxel size $=$ $1.0 \times 1.0 \times 1.0 \mathrm{~mm}^{3}$, field of view $=256 \mathrm{~mm}$ ).

\section{Behavioral Statistical Analyses}

The mean latencies of the correct manual responses and the proportion of errors were analyzed using a repeatedmeasures general linear model (GLM) with the within- subjects factors reward (high, low), task (arrow, word), trial type (repeat, switch), and the between-subjects factor DAT1 genotype. The first trial of every new block was excluded from the analysis (five trials per subject). The participants' number of Met alleles $(0,1$, or 2$)$ of the COMT Val ${ }^{108 / 158} \mathrm{Met}$ polymorphism was taken as covariate of non-interest in the between-subject analyses as the COMT polymorphism has been shown to interact in a linear manner with DAT1 on BOLD during reward anticipation (Dreher et al, 2009; but see, Yacubian et al, 2007). Specific effects were tested with paired $t$-tests. An effect was called significant when $P<0.05$. Estimates of effect size are given in partial eta squared $\left(\eta_{\mathrm{p}}^{2}\right)$. For the effects of reward receipt on subsequent task switching, we added the factor reward on the previous trial (no previous reward, previous reward) to the GLM.

\section{fMRI Statistical Analyses}

The pre-processed fMRI time series (see Supplementary Methods) were analyzed at the first level using an eventrelated approach in the context of the GLM. Our statistical model on the first (participant-specific) level considered the factors reward (high, low), task (arrow, word), trial type (repeat, switch), and feedback (correct-1 cent, correct-10 cents, error-0 cent). This resulted in 21 regressors of interest: 2 regressors for reward-cues, 8 regressors for task-cues (reward $\times$ task $\times$ trial type), 8 regressors for targets (reward $\times$ task $\times$ trial type), and 3 regressors for feedback ( 2 for correct responses and 1 for incorrect responses). All regressors of interest were modeled as an impulse response function (duration $=0$ ) convolved with a canonical hemodynamic response function. In addition, breaks (duration of $30 \mathrm{~s}$ ), feedback for misses ('too late - 0 cent' presented at the time of the response deadline), six motion parameters, and global signal changes (as indexed by segmented white matter and cerebral spinal fluid) were modeled as regressors of noninterest. High-pass filtering (128 s) was applied to the time series of the functional images to remove low-frequency drifts. Parameter estimates for all regressors were obtained by maximum-likelihood estimation, modeling temporal autocorrelation as an AR (autoregressive) (1) process.

Contrast images from the first level were entered into second level random-effect analyses to test consistent effects across participants. We calculated different two-sample $t$-tests on the basis of the contrasts specified at the first level, dividing the participants into two groups depending on their DAT1 genotype (9R carriers and 10R homozygotes). Furthermore, each of the second level designs included each participant's number of Met alleles $(0,1$, or 2) of the COMT Val ${ }^{108 / 158}$ Met polymorphism as covariate of non-interest. To measure reward anticipation and its interaction with DAT1 genotype, we included contrast images for high vs low reward-cues for each participant. Contrast images for high vs low task-cues were included in another two-sample $t$-test. To measure reward receipt and its interaction with DAT1 genotype, we included contrast images for positive feedback related to correct responses $v s$ negative feedback related to incorrect responses for each participant. To measure cognitive flexibility and its interaction with DAT1 genotype, we included contrast images for switch $v$ s repeat task-cues for each participant and we had a 
similar design for the switch $v s$ repeat targets. To measure the interaction between reward anticipation and cognitive flexibility and its interaction with DAT1 genotype, we included contrast images for the switch effect (switch $v s$ repeat) in low- vs high-reward trials for both task-cues and targets.

Statistical inference $(P<0.05)$ was performed at the cluster level, correcting for multiple comparisons over the search volume (the whole brain). The intensity threshold necessary to determine the cluster-level threshold was set at $P<0.001$, uncorrected.

Our a priori hypotheses allowed us to further investigate the effects of reward and its influence on cognitive flexibility in regions of interest (ROIs) in the striatum. We used the 'automated anatomical labeling' interface with SPM, which was developed by Tzourio-Mazoyer et al (2002), based on an anatomical parcellation of the spatially normalized single-subject high-resolution $\mathrm{T} 1$ volume provided by the MNI to select anatomically defined ROIs: left and right caudate nucleus (including ventromedial striatum, ie nucleus accumbens), and left and right putamen (see $3 \mathrm{a}$ and c). With MarsBaR (Brett et al, 2002), we extracted mean $\beta$ weights from all voxels in these ROIs for each individual first level design. These regionally averaged $\beta$ weights were analyzed using a repeated-measures GLM to investigate main and interaction effects during reward-cues, task-cues, targets, and feedback. Estimates of effect size are given in $\eta_{\mathrm{p}}^{2}$.

\section{Structural MRI Analysis}

As we were specifically interested in functional differences, we also investigated potentially confounding structural differences in the striatum between the two DAT1 genotype groups as measured with the anatomical scan. The procedure is described in Supplementary Methods.

\section{RESULTS}

As predicted, the degree of switch-related activity in the striatum varied as a function of reward magnitude and $D A T 1$ genotype. The DAT1 $(9 \mathrm{R} / 10 \mathrm{R}>10 \mathrm{R} / 10 \mathrm{R}) \times$ reward (high $>$ low) $\times$ trial type $($ switch $>$ repeat) interaction during targets was significant at a whole-brain corrected threshold in the left caudate nucleus (peak: $x=-12, y=18, z=8$, $T=5.33$; Figure $2 \mathrm{a}$ and $\mathrm{b}$ ), but in no other region. The pattern was bilateral when lowering the threshold to $P<0.005$ voxel level uncorrected for multiple comparisons, further strengthening its physiological plausibility. Analyses of data extracted from an anatomically defined ROI in the caudate nucleus confirmed that there was a significant three-way interaction during targets between reward, trial type, and DAT1 genotype in the left caudate nucleus $\left[F(1,18)=10.96, P=0.004, \eta_{\mathrm{p}}^{2}=0.38\right]$ and also revealed a strong trend in the right caudate nucleus $[F(1,18)=4.26$, $\left.P=0.054, \eta_{\mathrm{p}}^{2}=0.19\right]$. This three-way interaction was due to a significant simple reward $\times$ trial type interaction in the $9 \mathrm{R}$ carriers $\left[F(1,8)=13.12, P=0.007, \eta_{\mathrm{p}}^{2}=0.62\right]$, with greater switch-related activity on high-reward trials compared with low-reward trials (see also Supplementary Table S1; Supplementary Results). There was no significant simple two-way interaction in the 10R homozygotes $\left[F(1,10)=2.71, P>0.05, \eta_{\mathrm{p}}^{2}=0.21\right]$. During targets, there were no other interactions or main effects in these anatomical caudate nucleus ROIs.

The neural effect in the striatum was accompanied by a greater reward benefit in terms of error rates for the $9 \mathrm{R}$ carriers than the $10 \mathrm{R}$ homozygotes $[F(1,17)=4.86, P=0.04$, $\left.\eta_{\mathrm{p}}^{2}=0.22\right]$. Critically, this disproportionate reward benefit in the $9 \mathrm{R}$ carriers was restricted to the switch trials [reward by genotype: $F(1,18)=8.2, P=0.01, \eta_{\mathrm{p}}^{2}=0.33$, and did not extend to the repeat trials [reward by genotype: $F(1,17)<1$ ] [reward by trial type by genotype: $F(1,17)=4.62, P=0.046$, $\eta_{\mathrm{p}}^{2}=0.21$ ] (Figure 2d; see also Supplementary Table S1). The increased behavioral performance on switch trials (ie decreased error rates) on high-reward trials compared with low-reward trials in the $9 \mathrm{R}$ carriers paralleled the enhanced switch-related dorsomedial striatum activity on highreward trials compared with low-reward trials in those $9 \mathrm{R}$ carriers (Figure $2 \mathrm{~b}$ and $\mathrm{d}$ ). Thus, reward anticipation potentiated task switching and associated striatal activity in a DAT1-dependent manner.

Consistent with the hypothesized direction of information flow from ventral to dorsal striatal regions (Haber et al, 2000), the DAT1-dependent effect of reward on striatal activity during task switching was located in a region that was more dorsal and immediately adjacent to a ventromedial region of the striatum exhibiting a main effect of reward during reward anticipation (reward-cues; Figures 2a and 3a; Supplementary Table S2). This anticipation-related activity also depended on DAT1 genotype, as confirmed by analyses of data extracted from the anatomically defined ROI of the caudate nucleus (which includes the ventromedial striatum) [reward $\times D A T 1$ interaction-left: $F(1,18)=5.03, P=0.038$, $\eta_{\mathrm{p}}^{2}=0.22$; right: $\left.F(1,18)=4.34, P=0.052, \eta_{\mathrm{p}}^{2}=0.19\right]$. As shown in Figure 3b, 9R carriers showed a reward anticipation effect (high-low) [left: $F(1,8)=7.48$, $P=0.026, \quad \eta_{\mathrm{p}}^{2}=0.48$; right: $\quad F(1,8)=12.58, \quad P=0.008$, $\left.\eta_{\mathrm{p}}^{2}=0.61\right]$, whereas $10 \mathrm{R}$ homozygotes did to a much lesser extent [left: $F(1,10)<1$; right: $F(1,10)=1.5, \quad P>0.05$, $\left.\eta_{\mathrm{p}}^{2}=0.13\right]$. Furthermore, across subjects, anticipation-related activity in the ventromedial striatum correlated significantly and positively with the reward effect on switch-related activity in the more dorsomedial striatum (Pearson $r=0.49, t(18)=2.36, P=0.03$; Figure $2 c$ ).

Intriguingly, we also observed an interaction between $D A T 1$ genotype and the effect of reward during the receipt phase of the task. However, the DAT1 effect during reward receipt was in the opposite direction to that seen during reward-cues (Figure 3c). Moreover, it was located in the putamen, rather than in the caudate nucleus (feedback; Figure 3a; Supplementary Table S3). Thus, 9R carriers showed less activity in the putamen during the receipt of positive reward ( 1 or 10 cents for correct trials) relative to the receipt of negative reward (0 cent for incorrect trials) [left and right: $F(1,8)<1$ ] than did the $10 \mathrm{R}$ homozygotes [left: $F(1,10)=19.86, P=0.001, \eta_{\mathrm{p}}^{2}=0.67$; right: $F(1,10)=$ 15.00, $\left.P=0.003, \eta_{\mathrm{p}}^{2}=0.60\right]$. A direct comparison between the reward $\times D A T 1$ interaction effects in the caudate nucleus and the putamen during reward anticipation and reward receipt, respectively, confirmed this dissociation by revealing a significant three-way interaction between phase (anticipation in the caudate nucleus/receipt in the 
putamen), reward (magnitude and valence, respectively), and DAT1 genotype [left: $F(1,18)=9.67, P=0.006$, $\eta_{\mathrm{p}}^{2}=0.35$; right: $F(1,18)=8.53, P=0.009, \eta_{\mathrm{p}}^{2}=0.32$ ] (see also Supplementary Discussion). Interestingly, although reward anticipation potentiated task switching and associated striatal activity, the receipt of reward attenuated subsequent task switching as shown by increased switch costs after positive feedback relative to negative feedback in terms of both response times [previous reward $\times$ trial type interaction: $F(1,11)=5.31, P=0.042, \eta_{\mathrm{p}}^{2}=0.33$ ] and error rates [previous reward $\times$ trial type interaction: $F(1,15)=$ 4.81, $P=0.044, \quad \eta_{\mathrm{p}}^{2}=0.24$ ] (Supplementary Figure S1; Supplementary Discussion).

To summarize, we observed striatal activity during reward anticipation, task switching, and reward receipt. However, there was a region-specific dissociation between the effects of DAT1 on striatal activity depending on task period. First, the $9 \mathrm{R}$ carriers - with presumably more striatal dopamine-showed greater reward (magnitude)related activity in the ventromedial striatum than did the $10 R$ homozygotes during reward anticipation. Second, 9R carriers also exhibited a greater effect of reward on switch costs, as well as on switch-related activity in the dorsomedial striatum during task switching. Finally, the 9R carriers showed less reward (valence)-related activity in the lateral striatum than did the $10 R$ homozygotes during reward receipt (Figure 3 ).

\section{COMT Genotype}

We were specifically interested in striatal and not frontal dopamine. Therefore, we corrected all effects of DAT1 for the COMT Val ${ }^{108 / 158}$ Met (rs4680) genotype by using COMT genotype as a covariate (see Materials and methods). Note that all effects of DAT1 were significant even if they were not corrected for COMT. Furthermore, there were no effects of COMT genotype on task-related activity by itself.

\section{Structural Imaging Data}

Using the anatomical data, we tested whether structural differences in striatal volumes (as percentage of total brain volume) between the DAT1 groups could account for the functional differences in brain activity we observed between a

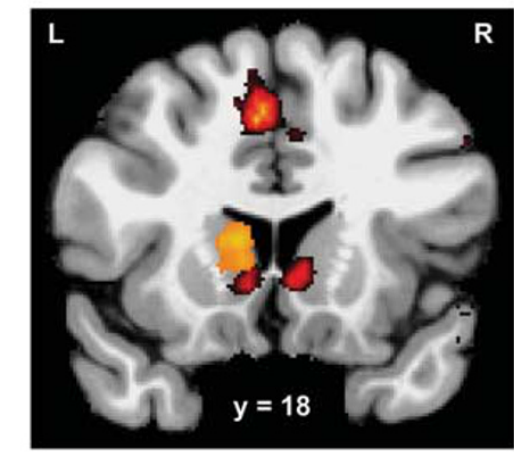

C

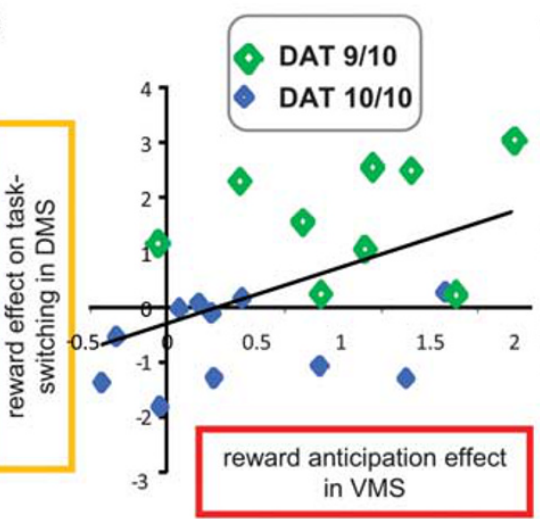

b
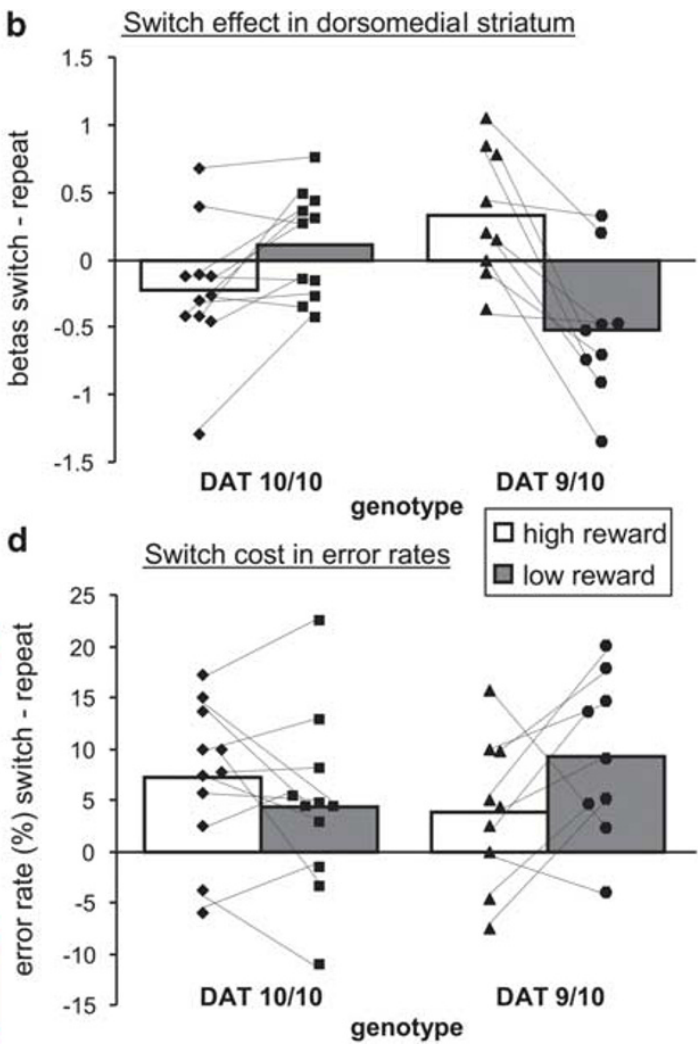

Figure 2 Effects of dopamine on task switching as a function of reward. (a) Coronal section showing increased left caudate nucleus activity for the threeway interaction on the targets between reward anticipation, task switching, and DATI genotype in orange, and the main effect of reward anticipation during reward-cues in red (voxel-level cutoff at $P<0.001$, uncorrected). $L=$ left; $R=$ right. (b) For illustration purposes, we plotted the switch effect (switch - repeat targets) from the suprathreshold voxels in the dorsomedial striatum. Bars represent the average across subjects; symbols represent individual data points; dotted lines connect the data points belonging to the same subject. All 9R carriers showed larger switch-related activity in the dorsomedial striatum when anticipating high reward relative to the anticipation of low reward. (c) The dopamine-modulated effect of anticipated reward on task switching in the dorsomedial striatum (DMS) correlated significantly with the reward anticipation effect in the ventromedial striatum (VMS) ( $r=0.49$, $P=0.03$ ), with the $9 R$ carriers (DAT 9/9) showing both increased activity in dorsomedial striatum as well as ventromedial striatum compared with the IOR homozygotes (DAT I0//0). Note that the plotted data were extracted from the suprathreshold voxels representing the three-way interaction between genotype, reward, and task switching and the main effect of reward anticipation, respectively. (d) The error switch cost (switch-repeat trials). Bars represent the average across subjects; symbols represent individual data points; dotted lines connect the data points belonging to the same subject. Almost all 9R carriers showed a smaller switch cost when anticipating high reward relative to the anticipation of low reward. 
a Reward anticipation and reward receipt in striatum

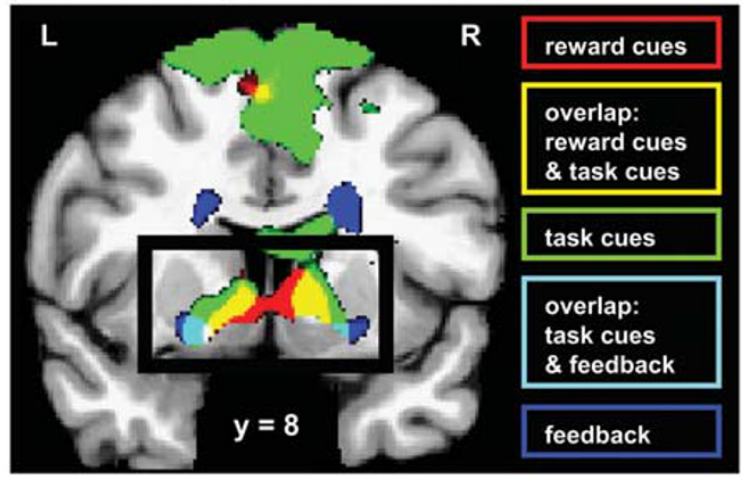

b Reward anticipation effect in anatomical left caudate ROI
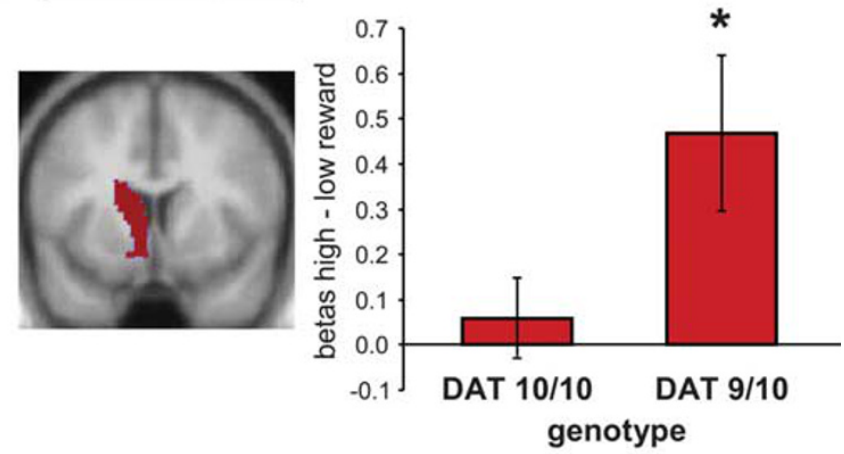

C Reward receipt effect in anatomical left putamen ROI

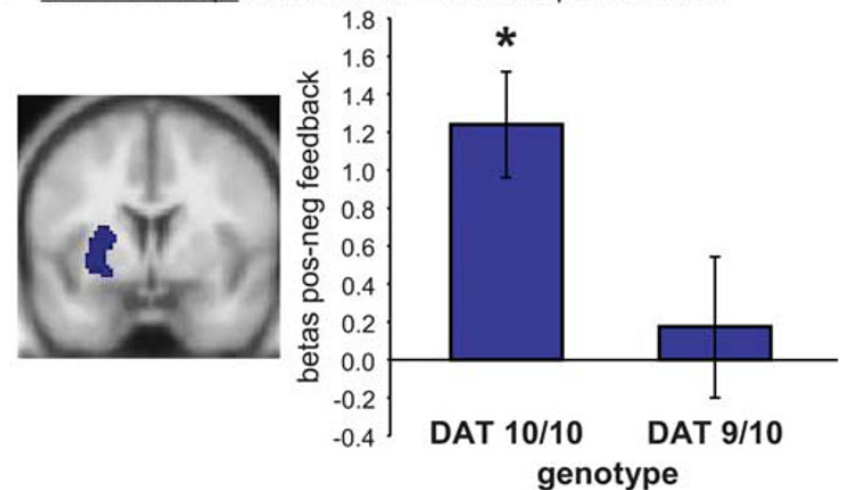

Figure 3 Reward anticipation and reward receipt in the striatum. (a) Whole-brain results for the main effects of reward reveal a medial-tolateral gradient as a function of task phase in ventral striatum: The effect of reward anticipation during reward-cues (in red) was largest in the ventromedial part of the striatum. The effect of reward anticipation during task-cues (in green) is observed more laterally in the striatum. Finally, the effect of reward receipt (in dark blue) was largest in the most lateral parts of the ventral striatum (voxel-level cutoff at $P<0.00$ I, uncorrected). $\mathrm{L}=$ left; $\mathrm{R}=$ right. (b) The reward anticipation effect in the anatomically defined (left) caudate nucleus is plotted for the IOR homozygotes (DAT $10 / 10$ ) and the 9 R carriers (DAT 9/I0). The 9R carriers (with presumably more striatal dopamine), but not the IOR homozygotes, showed a significant reward anticipation effect (see *) in this caudate $\mathrm{ROI}$, which includes the nucleus accumbens. Error bars represent standard errors of the differences between high- and low-reward-cues. (c) The reward receipt effect in the anatomically defined (left) putamen is plotted for the IOR homozygotes (DAT I0//0) and the 9R carriers (DAT 9/I0). The IOR homozygotes (with presumably less striatal dopamine), but not the $9 R$ carriers, showed a significant reward receipt effect (see *) in this putamen ROI. Error bars represent standard errors of the differences (SED) between positive feedback and negative feedback. the groups. There were no differences between the groups in the volumes of the nucleus accumbens (left: $t(18)=0.08$, $P>0.9$; right: $t(18)=-0.1, P>0.8$ ), caudate nucleus (left: $t(18)=-0.7, P>0.4$; right: $t(18)=-0.4, P>0.6)$, putamen (left: $t(18)=1.5, P>0.1$; right: $t(18)=0.7, P>0.5$ ), or total striatal volume $(t(18)=0.4, P>0.6)$. Thus, it is unlikely that the functional differences we observed between the DAT1 groups are caused by volumetric differences in the striatum.

\section{DISCUSSION}

The present data establish the importance of dopamine in the human striatum for the potentiation of cognitive flexibility by reward anticipation. Such a role for dopamine in the interface between motivational and cognitive control processes had previously been hypothesized based on work with experimental animals (Baldo and Kelley, 2007) and neuroanatomical evidence for spiraling connections between the midbrain and the striatum (Haber, 2003; Haber et al, 2000). Here, we provide the first evidence for this hypothesis in humans, both in terms of behavior and in terms of striatal activity. The 9R carriers exhibited greater effects of reward on behavioral switch costs as well as on switch-related striatal activity than did 10R homozygotes. These findings reinforce the importance of the striatum for task switching (Cools, 1980; Cools et al, 2004; Leber et al, 2008; Lyon and Robbins, 1975; Oades, 1985), a process previously associated almost exclusively with the PFC (Sakai, 2008). Specifically, we show that the degree to which task switching is influenced by motivational state depends on genetic variation in the DAT, which is most abundant in the striatum, and is accompanied by modulation of activity in the striatum only.

Accumulating neural and behavioral data suggest that behavior can be controlled by multiple systems, including on the one hand, a flexible, reward-oriented controller that is directly sensitive to changes in motivational state and which has been associated with the PFC and medial striatum, and, on the other hand, an inflexible, habitual controller, which has been associated with the dorsolateral striatum (Daw et al, 2005; Dayan, 2008; Dickinson and Balleine, 2002). According to these theoretical models, striatal dopamine is implicated primarily in the dorsolateral habitual controller. This work suggests that such theoretical models might be revised, and extended by including an important role for dopamine in modulating the medial striatum and flexible, motivational state-dependent cognitive control processes.

The mechanism by which the striatum controls cognitive flexibility has been elucidated in the domain of working memory. That is, computational modeling work has suggested a gating function of the striatum (Frank et al, 2001). More specifically, the striatum can selectively facilitate the updating of task-relevant representations in the PFC, analogous to the gating of appropriate motor plans to the motor cortex (through the thalamus) in the motor parts of the striatum (Mink, 1996). Here, we show experimentally, with a task-switching paradigm, that the striatum is indeed necessary for gating or flexibly updating of goal-relevant representations in the cognitive domain.

Dopamine transmission has been shown to influence brain structure and plasticity (Chakos et al, 1995; Selemon 
et al, 1999). Accordingly, it might be argued that the effects of genetic variation in the DAT reflect inter-individual differences in brain morphometry. However, in this study in young healthy volunteers, we could not find volume differences in striatal structures between the two genotype groups. Thus, it is unlikely that our functional findings are caused by structural differences between the groups. Several in vitro and in vivo studies have shown that the $9 \mathrm{R}$ allele is associated with reduced expression of DAT1 and reduced DAT binding (eg Fuke et al, 2001; Heinz et al, 2000; Mill et al, 2002; VanNess et al, 2005), thus likely increased DA transmission by reducing synaptic DA clearance rates (although there is also some controversy; van Dyck et al, 2005). Therefore, we argue that the effects reflect individual differences in dopamine transmission in the striatum.

The novel finding that the effect of reward on task switching depended on DAT1 genotype was obtained in the context of a replication of prior findings. Specifically, we replicated previous observations that $9 \mathrm{R}$ carriers exhibit enhanced reward-related activity in the ventromedial striatum (Dreher et al, 2009; Forbes et al, 2009). Such replication of genetic imaging findings with this relatively small sample size adds significant value by itself, but also lends credence to our critical novel three-way interaction effect with task switching. Nonetheless, these preliminary findings should be interpreted with caution till replicated in an independent sample. The present task-phase-dependent genotype effects might help to resolve some apparently conflicting results from recent genetic imaging studies that focused on polymorphisms in the gene coding for the dopamine D2 receptor (DRD2). In these studies, genetically determined reduced DRD2 receptor density has been found to be accompanied by either increased striatal activity during reward anticipation (Kirsch et al, 2006) or decreased activity during reward receipt (Cohen et al, 2007; Klein et al, 2007). The present results suggest that these apparently conflicting effects in participants with genetically reduced D2 receptor density (which presumably leads to enhanced dopamine synthesis and release through attenuation of D2dependent autoregulation; Cooper et al, 2003; Forbes et al, 2009) reflect the fact that effects were obtained during distinct task phases.

Indeed, in this study, the facilitatory effect of the DAT1 polymorphism on striatal activity was restricted to the reward anticipation and task-switching phases of the task. In contrast to anticipation-related activity, we found that receipt-related activity was actually reduced in the $9 \mathrm{R}$ carriers. Critically, these effects were seen in medial and lateral regions of the striatum, respectively. The regiondependent effect of DAT1 genotype as a function of task phase suggests that dopamine's effect on medial striatal function and associated reward anticipation might be accompanied by an opponent effect on lateral striatal function and associated consummatory reward processing. The pattern is reminiscent of those observed in studies with rodents, in which effects of manipulating dopamine in the ventromedial striatum are more readily observed on preparatory than on consummatory behaviors (Robbins and Everitt, 1992; Salamone et al, 2007). Moreover, some studies have reported that consummatory responses can be attenuated by increases in dopamine (eg after amphetamine administration, which can have anorexic effects; eg Bakshi and Kelley, 1991; but see Kelley et al, 1989), and enhanced by decreases in dopamine (eg by 6-OHDA lesions; see Baldo and Kelley, 2007; Koob et al, 1978). Instead, it has been postulated that opioids directly modulate consummatory responses (Berridge and Robinson, 1998). The present genetic imaging study suggests that such opposite effects of dopamine on preparatory and consummatory reward processes might also be present in humans. As pointed out in Introduction, one of the characteristic features of preparatory responding is its flexible nature and such flexible preparatory behavior contrasts with the relatively inflexible, stereotypical behaviors required for the adequate commerce of reward (Baldo and Kelley, 2007; Craig, 1918). In keeping with this observation, we found that switch costs were reduced when subjects anticipated reward, but actually enhanced when subjects had just received reward (see Supplementary Figure S1). The current observation that the opposite genotype effects during anticipation and receipt differed between regions is in line with the proposal, based again on work with rodents, that preparatory and consummatory processes implicate distinct regions of the striatum (Robbins and Everitt, 1992; Voorn et al, 2004).

Previous genetic imaging studies have found interaction effects of DAT1 and COMT (rs4680) genotype on rewardrelated striatal activity (Dreher et al, 2009; Yacubian et al, 2007). The division of alleles across groups was not such that we could separately analyze the effects of COMT genotype and its interaction with DAT1. However, we did take COMT genotype into account as a covariate and, hence, were able to show that our DAT1 effects were independent of COMT genotype. Thus, although this lack of effect of COMT should be replicated in future studies, it does strengthen our hypothesis that the effect of anticipatory reward on task switching is likely mediated by striatal not frontal dopamine. Recently, it was shown with fMRI that executive control and other cognitive processes in humans were modulated by monetary reward (Adcock et al, 2006; Kouneiher et al, 2009; Krawczyk et al, 2007; Wittmann et al, 2005). Here, we show similar evidence for executive control processes in the human striatum and, moreover, we show the involvement of striatal dopamine in this interface between motivational and cognitive control.

To conclude, our genetic imaging study indicates a critical role for human striatal dopamine in reward anticipation and its influence on cognitive flexibility. In doing so, our results provide direct support for previously hypothesized neurochemical mechanism for the interface between motivation and cognition. The finding extends classic observations that behavioral flexibility depends on striatal dopamine (Cools, 1980; Lyon and Robbins, 1975; Oades, 1985) to the domain of human motivation and cognition, thus drawing attention to the importance of striatal dopamine for higher-order cognitive control processing.

\section{ACKNOWLEDGEMENTS}

We thank Carline van der Pol for the structural MRI analysis, Marlies Naber and Johanne Groothuismink for genotyping, and Sabine Kooijman for collecting the samples. This work was supported by a VICI grant of the 
Netherlands Organisation for Scientific Research to AR; $\mathrm{RCH}$ was supported by a grant from the Alkemade-Keuls foundation.

\section{DISCLOSURE}

The authors declare that, except for income received from primary employers, no financial support or compensation has been received from any individual or corporate entity over the past 3 years for research or professional service and there are no personal financial holdings that could be perceived as constituting a potential conflict of interest.

\section{REFERENCES}

Aarts E, Roelofs A, van Turennout M (2008). Anticipatory activity in anterior cingulate cortex can be independent of conflict and error likelihood. J Neurosci 28: 4671-4678.

Aarts E, Roelofs A, van Turennout M (2009). Attentional control of task and response in lateral and medial frontal cortex: brain activity and reaction time distributions. Neuropsychologia 47: 2089-2099.

Adcock RA, Thangavel A, Whitfield-Gabrieli S, Knutson B, Gabrieli JD (2006). Reward-motivated learning: mesolimbic activation precedes memory formation. Neuron 50: 507-517.

Bakshi VP, Kelley AE (1991). Dopaminergic regulation of feeding behavior: II. Differential effects of amphetamine microinfusion into three striatal subregions. Psychobiology 19: 233-242.

Baldo BA, Kelley AE (2007). Discrete neurochemical coding of distinguishable motivational processes: insights from nucleus accumbens control of feeding. Psychopharmacology (Berl) 191: 439-459.

Berridge KC, Robinson TE (1998). What is the role of dopamine in reward: hedonic impact, reward learning, or incentive salience? Brain Res Brain Res Rev 28: 309-369.

Brett M, Anton JL, Valabregue R, Poline JB (eds) (2002). Region of interest analysis using an SPM toolbox. 8th International Conference on Functional Mapping of the Human Brain. NeuroImage: Sendai, Japan.

Chakos MH, Lieberman JA, Alvir J, Bilder R, Ashtari M (1995). Caudate nuclei volumes in schizophrenic patients treated with typical antipsychotics or clozapine. Lancet 345: 456-457.

Cohen MX, Krohn-Grimberghe A, Elger CE, Weber B (2007). Dopamine gene predicts the brain's response to dopaminergic drug. Eur J Neurosci 26: 3652-3660.

Cools AR (1980). Role of the neostriatal dopaminergic activity in sequencing and selecting behavioural strategies: facilitation of processes involved in selecting the best strategy in a stressful situation. Behav Brain Res 1: 361-378.

Cools R, Clark L, Robbins TW (2004). Differential responses in human striatum and prefrontal cortex to changes in object and rule relevance. J Neurosci 24: 1129-1135.

Cooper JC, Bloom FE, Roth RH (2003). The Biochemical Basis of Neuropharmacology, 8th edn. Oxford University Press: Oxford.

Craig W (1918). Appetites and aversions as constituents of instincts. Biol Bull 34: 91-107.

Daw ND, Niv Y, Dayan P (2005). Uncertainty-based competition between prefrontal and dorsolateral striatal systems for behavioral control. Nat Neurosci 8: 1704-1711.

Dayan P (2008). The role of value systems in decision making. In: Engel C, Singer W (eds). Better Than Conscious? Decision Making, the Human Mind, and Implications for Institutions. MIT Press: Cambridge, MA, pp 51-70.

Dickinson A, Balleine BW (2002). The role of learning in motivation. In: Gallistel CR (ed). Stevens' Handbook of Experi- mental Psychology Vol. 3: Learning, Motivation and Emotion, 3rd edn. Wiley: New York. Vol 3, pp 497-533.

Dreher JC, Kohn P, Kolachana B, Weinberger DR, Berman KF (2009). Variation in dopamine genes influences responsivity of the human reward system. Proc Natl Acad Sci USA 106: 617-622.

Forbes EE, Brown SM, Kimak M, Ferrell RE, Manuck SB, Hariri AR (2009). Genetic variation in components of dopamine neurotransmission impacts ventral striatal reactivity associated with impulsivity. Mol Psychiatry 14: 60-70.

Frank MJ, Loughry B, O’Reilly RC (2001). Interactions between frontal cortex and basal ganglia in working memory: a computational model. Cogn Affect Behav Neurosci 1: 137-160.

Fuke S, Suo S, Takahashi N, Koike H, Sasagawa N, Ishiura S (2001). The VNTR polymorphism of the human dopamine transporter (DAT1) gene affects gene expression. Pharmacogenomics $J$ 1: $152-156$.

Haber SN (2003). The primate basal ganglia: parallel and integrative networks. J Chem Neuroanat 26: 317-330.

Haber SN, Fudge JL, McFarland NR (2000). Striatonigrostriatal pathways in primates form an ascending spiral from the shell to the dorsolateral striatum. J Neurosci 20: 2369-2382.

Heinz A, Goldman D, Jones DW, Palmour R, Hommer D, Gorey JG et al (2000). Genotype influences in vivo dopamine transporter availability in human striatum. Neuropsychopharmacology 22: 133-139.

Ikemoto S, Panksepp J (1999). The role of nucleus accumbens dopamine in motivated behavior: a unifying interpretation with special reference to reward-seeking. Brain Res Brain Res Rev 31: 6-41.

Kelley AE, Gauthier AM, Lang CG (1989). Amphetamine microinjections into distinct striatal subregions cause dissociable effects on motor and ingestive behavior. Behav Brain Res 35: 27-39.

Kirsch P, Reuter M, Mier D, Lonsdorf T, Stark R, Gallhofer B et al (2006). Imaging gene-substance interactions: the effect of the DRD2 TaqIA polymorphism and the dopamine agonist bromocriptine on the brain activation during the anticipation of reward. Neurosci Lett 405: 196-201.

Klein TA, Neumann J, Reuter M, Hennig J, von Cramon DY, Ullsperger M (2007). Genetically determined differences in learning from errors. Science 318: 1642-1645.

Koob GF, Riley SJ, Smith SC, Robbins TW (1978). Effects of 6hydroxydopamine lesions of the nucleus accumbens septi and olfactory tubercle on feeding, locomotor activity, and amphetamine anorexia in the rat. J Comp Physiol Psychol 92: 917-927.

Kouneiher F, Charron S, Koechlin E (2009). Motivation and cognitive control in the human prefrontal cortex. Nat Neurosci 12: 939-945.

Krawczyk DC, Gazzaley A, D’Esposito M (2007). Reward modulation of prefrontal and visual association cortex during an incentive working memory task. Brain Res 1141: 168-177.

Leber AB, Turk-Browne NB, Chun MM (2008). Neural predictors of moment-to-moment fluctuations in cognitive flexibility. Proc Natl Acad Sci USA 105: 13592-13597.

Logan GD, Bundesen C (2003). Clever homunculus: is there an endogenous act of control in the explicit task-cuing procedure? J Exp Psychol Hum Percept Perform 29: 575-599.

Lyon M, Robbins TW (1975). The action of central nervous system drugs: a general theory concerning amphetamine effects. In: Essman W (ed). Current Developments in Psychopharmacology. Spectrum publications: New York. Vol 2, pp 80-163.

Mill J, Asherson P, Browes C, D'Souza U, Craig I (2002). Expression of the dopamine transporter gene is regulated by the $3^{\prime}$ UTR VNTR: evidence from brain and lymphocytes using quantitative RT-PCR. Am J Med Genet 114: 975-979.

Mink JW (1996). The basal ganglia: focused selection and inhibition of competing motor programs. Prog Neurobiol 50: 381-425. 
Mogenson GJ, Jones DL, Yim CY (1980). From motivation to action: functional interface between the limbic system and the motor system. Prog Neurobiol 14: 69-97.

Monsell S (2003). Task switching. Trends Cogn Sci 7: 134-140.

Nauta WJ, Smith GP, Faull RL, Domesick VB (1978). Efferent connections and nigral afferents of the nucleus accumbens septi in the rat. Neuroscience 3: 385-401.

Oades RD (1985). The role of noradrenaline in tuning and dopamine in switching between signals in the CNS. Neurosci Biobehav Rev 9: 261-282.

Robbins TW, Everitt BJ (1992). Functions of dopamine in the dorsal and ventral striatum. Semin Neurosci 4: 119-127.

Roelofs A, van Turennout M, Coles MG (2006). Anterior cingulate cortex activity can be independent of response conflict in stroop-like tasks. Proc Natl Acad Sci USA 103: 13884-13889.

Sakai K (2008). Task set and prefrontal cortex. Annu Rev Neurosci 31: 219-245.

Salamone JD, Correa M, Farrar A, Mingote SM (2007). Effort-related functions of nucleus accumbens dopamine and associated forebrain circuits. Psychopharmacology (Berl) 191: 461-482.

Schultz W (2002). Getting formal with dopamine and reward. Neuron 36: 241-263.

Selemon LD, Lidow MS, Goldman-Rakic PS (1999). Increased volume and glial density in primate prefrontal cortex associated with chronic antipsychotic drug exposure. Biol Psychiatry 46: 161-172.

Tzourio-Mazoyer N, Landeau B, Papathanassiou D, Crivello F, Etard O, Delcroix N et al (2002). Automated anatomical labeling of activations in SPM using a macroscopic anatomical parcellation of the MNI MRI single-subject brain. Neuroimage 15: 273-289.

van Dyck CH, Malison RT, Jacobsen LK, Seibyl JP, Staley JK, Laruelle $\mathrm{M}$ et al (2005). Increased dopamine transporter availability associated with the 9-repeat allele of the SLC6A3 gene. J Nucl Med 46: 745-751.

VanNess SH, Owens MJ, Kilts CD (2005). The variable number of tandem repeats element in DAT1 regulates in vitro dopamine transporter density. BMC Genet 6: 55.

Voorn P, Vanderschuren LJ, Groenewegen HJ, Robbins TW, Pennartz CM (2004). Putting a spin on the dorsal-ventral divide of the striatum. Trends Neurosci 27: 468-474.

Wittmann BC, Schott BH, Guderian S, Frey JU, Heinze HJ, Duzel E (2005). Reward-related FMRI activation of dopaminergic midbrain is associated with enhanced hippocampus-dependent long-term memory formation. Neuron 45: 459-467.

Yacubian J, Sommer T, Schroeder K, Glascher J, Kalisch R, Leuenberger B et al (2007). Gene-gene interaction associated with neural reward sensitivity. Proc Natl Acad Sci USA 104: 8125-8130.

Supplementary Information accompanies the paper on the Neuropsychopharmacology website (http://www.nature.com/npp) 